\title{
Extending anisotropic operators to recover smooth shapes
}

\author{
Debora Gil*, Petia Radeva \\ Computer Vision Center (CVC), Edifici O, Campus UAB 08193 Bellaterra, Barcelona, Spain
}

Received 18 March 2003; accepted 15 December 2004

Available online 26 January 2005

\begin{abstract}
Anisotropic differential operators are widely used in image enhancement processes. Recently, their property of smoothly extending functions to the whole image domain has begun to be exploited. Strong ellipticity of differential operators is a requirement that ensures existence of a unique solution. This condition is too restrictive for operators designed to extend image level sets: their own functionality implies that they should restrict to some vector field. The diffusion tensor that defines the diffusion operator links anisotropic processes with Riemmanian manifolds. In this context, degeneracy implies restricting diffusion to the varieties generated by the vector fields of positive eigenvalues, provided that an integrability condition is satisfied. We will use that any smooth vector field fulfills this integrability requirement to design line connection algorithms for contour completion. As application we present a segmenting strategy that assures convergent snakes whatever the geometry of the object to be modelled is.
\end{abstract}

(c) 2004 Elsevier Inc. All rights reserved.

Keywords: Contour completion; Functional extension; Differential operators; Riemmanian manifolds; Snake segmentation

\footnotetext{
This work is partially supported by the "Ministerio de Ciencia y Tecnologia" Grant TIC2000-1635C04-04.

* Corresponding author.

E-mail addresses: debora@cvc.uab.es (D. Gil), petia@cvc.uab.es (P. Radeva).
} 


\section{Introduction}

The fact that solutions to a diffusion equation are infinitely differentiable has been largely used in image enhancement and restoration. However, the capability of smoothly extending functions to the whole image domain has been hardly exploited. Only in recent years some authors have used this property to extend vector fields [22] and, to some extend, to fill-in gaps in the image [2]. Object disocclusion, image restoration (in-painting), shape reconstruction, splines interpolation or, even, segmentation by snakes are some of the different fields in image processing that address the issue of line connection.

Object disocclusion and image in-painting focus on filling in gaps in an image in such a way that level lines arriving at gaps boundaries are smoothly prolonged inside. Following the Gestalt principle of good continuation, recent techniques $([2,5,17,18,12])$ base on the way humans join unconnected curves to reconstruct the underlying shape. According to psychophysicist studies [14], the curves that reconstruct objects should minimize an energy functional involving length and curvature. To such purpose they seek for the function, which corresponds to the reconstructed image, that achieves a simultaneous minimum for all its level sets. The continuous variational problem includes a fourth order term [5] that troubles the numeric implementation. Solutions, up to our knowledge, include simplification of the functional, specific numeric algorithms [5], approximations to the solution [17] or the introduction [2] of an extra function to be minimized.

The second group of techniques (shape reconstruction and segmentation) deal with single contour completion. Shape recovery aims at obtaining a smooth model of shapes from a unconnected set of points, meanwhile object image segmentation must face connecting a set of points that lie on the contour of the objects of interest. An efficient way of modelling uncompleted shapes is by means of a snake $[3,4,15]$. Snakes or active contours correspond to minimums of an energy functional. Classic snakes [15] and geodesic snakes [3,4] are the two most popular energy designs recognized by the image processing community. Classic snakes produce smooth models of shapes but their convergence strongly depends on the definition of the external energy (commonly, distance or edge based map) and the snake parameterization. In spite of the improvements that the gradient vector flow $(\mathrm{GVF}) /$ generalized gradient vector flow (GGVF) [22] provides, classic snake convergence to concave curves is still poor. In the case of geodesic snakes, the internal and external energies join to produce a functional that measures the length of the snake in a Riemmanian manifold with the image gradient external energy as metric. Their level sets formulation [20] ensures convergence to an arbitrary number of objects. However, minimizing curve length leads to piece wise linear interpolation of the set of points and shock formation during the level sets evolution makes this formulation computationally expensive [19]. Moreover, balloon forces used to increase convergence to contours might push the snake into the object contour in the presence of large gaps.

In this paper, we address level sets completion for segmenting purposes. We consider the problem of image segmentation as the recovery of smooth models from a set of points, usually unconnected, on the objects of interest. The problem can be split in 
two main steps: interpolation of the curve segments (shape restoration) and computation of a smooth representation of shapes as compact as possible (e.g., by parametric snakes). The goal of this paper is to develop an anisotropic contour closing (ACC) technique as a tool for shape restoration in a first step for image segmentation.

We separate the problem of level sets completion into computing a smooth vector field on the image domain representing the unit tangent to the set of curves to be reconstructed and then integrate it to obtain the corresponding level sets. Like in disocclusion and in-painting techniques, we also suggest a level sets formulation to simultaneously integrate all curves. The novelty consists in embedding the integration problem into the framework of functional extension by means of second order differential operators given in divergence form via a symmetric diffusion tensor [7]. In the context of level sets completion the tensor should degenerate/cancel in the gradient direction. In the present paper, we perform a study of diffusion tensors from the point of view of differential geometry which provides us with a criterion to decide when a degenerate tensor still produces solvable PDEs. This reduces the completion problem to the definition of a smooth vector field representing the level sets to be extended, which brings our technique very close to the stochastic completion fields of [12]. Our second contribution is a fast reliable extension of vector fields based on the properties of the structure tensor [13]. Our experiments illustrate robustness of ACC to handle reconstruction of T-junctions and corners, as well as its capability of recovering illusory contours. Contours interpolated with ACC are, like geodesic snakes, given in an implicit representation as level sets of an image. For an explicit compact encoding parametric B-snakes are a sensible choice. To ensure convergence to shapes we use a previously developed technique [9] which in combination with ACC yields a segmenting strategy that successfully copes with the poor convergence of snakes to concave shapes. Results presented in the second part of the experimental Section show that the convergence rate compares to that of geodesic snakes, performing even better in noisy images and real images with textured backgrounds.

The paper is organized as follows. Section 2 deals with the general setting of functional extension, as well as, exposes the geometric theory about restricted diffusion tensors. A throughout description of ACC is given in Section 3, including the different approaches to extension of vector fields in Section 3.1. The experimental Section 4 presents results showing accuracy of shapes restored with ACC in Section 4.1 and efficiency of the segmentation algorithm compared to geodesic snakes in Section 4.2. Conclusions and future lines of research are exposed in Section 5.

\section{Restricted anisotropic operators}

Any second order partial differential operator, namely $L$, defines, both, a diffusion process:

$$
u_{t}=L u \quad \text { with } \quad u(x, 0)=u_{0}(x)
$$

and a functional extension:

$$
L u=0 \quad \text { with } \quad u_{\mid \partial \Omega}=f
$$


for $\partial \Omega$ an $n-1$ dimensional manifold on $\mathbb{R}^{n}$ and $f$ the function on $\partial \Omega$ we want to extend. Solutions to (1) are computed by means of the evolution diffusion equation:

$$
u_{t}=L u \quad \text { with } \quad u_{\mid \partial \Omega}=f
$$

as they correspond to its steady states.

In all cases, existence and uniqueness (in the weak sense) of smooth solutions is guaranteed if $L$ is strongly elliptic [7,23], that is, when it defines a scalar product on some functional space. However, extensions focused on level sets continuation should restrict diffusion to a vector field representing the level sets of the solution. Thus, the hypothesis of strong ellipticity is relaxed and we must tackle with operators that degenerate on some vector fields.

Let us give some geometric requirements over the non null space of $L$ that ensure existence of solutions in the case of an operator given in divergence form. In this case, we have that:

$$
L u=\operatorname{div}(J \nabla u)
$$

for $J$ a symmetric (semi) positive defined tensor (quadratic form). Strong ellipticity means that all eigenvalues of $J$ are strictly positive, meanwhile tensors having a null space (kernel) of positive dimension will produce degenerate operators. Because any symmetric (semi) positive defined tensor defines a scalar product/metric, we will embed elliptic operators into the framework of Riemmanian geometry and use tools of differential geometry to analyze solvability of degenerate operators. Details regarding results on differential geometry can be found in [21].

Let $\left(\mathbb{R}^{n}, g\right)$ be a Riemmanian manifold with the metric, $g$, given by a tensor $J$. Since the matrix $J$ is symmetric, it diagonalizes [16] (considered as linear map) in an orthonormal basis that completely describes the metric. If $Q$ is the coordinate change, then we have that, as bilinear form, $J=Q \Lambda Q^{t}$, for $\Lambda$ the eigenvalue matrix. In this context, we can talk about isotropic diffusion (equal eigenvalues), anisotropic diffusion (distinct and strictly positive eigenvalues) and restricted diffusion ${ }^{1}$ (in the case of some null eigenvalues), respectively. That is, the restricted diffusion is given by a diffusion tensor, $\tilde{J}$, defined by the following eigenvalue matrix:

$$
\Lambda=\left(\begin{array}{ccc|c}
\lambda_{1} & \cdots & 0 & \\
\vdots & \ddots & \vdots & 0 \\
0 & \cdots & \lambda_{m} & \\
\hline & 0 & & 0
\end{array}\right)
$$

Indeed we will only consider the homogeneous case $\lambda_{i}=1$, for all $i$. Let us determine under what conditions a degenerated metric makes sense. Let $\xi_{1}, \ldots, \xi_{k}$ be the eigenvectors of positive norm and denote by $\mathscr{D}=\left\langle\xi_{1}, \ldots, \xi_{k}\right\rangle$ the vector space (distribution) they generate. If such vector space was the tangent space to a sub manifold of $\mathbb{R}^{n}$ (integral variety of $\mathscr{D}$ ), then the metric $\tilde{J}$ would be the projection onto its tangent

\footnotetext{
${ }^{1}$ The word restricted applies to the the fact that diffusion restricts to the manifolds generated by the vectors of positive eigenvalues.
} 
space. Consequently a diffusion process governed by $\tilde{J}$ would not take place in the whole space $\mathbb{R}^{n}$ but just on the integral manifolds, namely $M$, of the distribution. We claim that this integrability condition and compactness are the only requirements for a unique solution to (1) given by a restricted diffusion tensor $\tilde{J}$ :

Proposition 2.1. Let $\tilde{J}$ be a degenerate metric tensor on $\mathbb{R}^{n}$. Assume that the distribution $\mathscr{D}=\left\langle\xi_{1}, \ldots, \xi_{k}\right\rangle$ generated by the eigenvector fields of positive eigenvalues is integrable. Let $f=f\left(x_{1}, \ldots, x_{n}\right)$ be a smooth function defined on a sub-manifold $\partial \Omega$ enclosing a compact volume. Then there exists a unique solution to:

$$
\operatorname{div}(\tilde{J} \nabla u)=0 \text { with } u_{\mid \partial \Omega}=f
$$

which is as smooth as the boundary function $f$.

Proof. The basic idea is that the differential operator restricts to a elliptic operator on each of the integral manifolds, $M$, defined by the distribution $\mathscr{D}$. By a classic result on heat operators on manifolds [6], we have that it exists a unique smooth solution that extends $f_{\mid \partial \Omega} \cap M$ to the whole leave $M$. The fact that by Frobenius Theorem these manifolds foliate (i.e., decompose the entire space into layers) the space yield the existence of a unique solution to the higher dimensional problem [10].

The integrability condition ensuring existence of solutions is a standard result on differential manifolds known as the Frobenius Theorem [21]. The latter states that there exist integral manifolds for a distribution $\mathscr{D}$ provided that the vectors generating $\mathscr{D}$ fulfill an algebraic condition ( $\mathscr{D}$ involutive). That is, a local condition on the potential tangent spaces ensures that there will exist manifolds having $\mathscr{D}$ as tangent space. To be precise, the integrability condition is given in terms of the Lie bracket of $\xi_{1}, \ldots, \xi_{k}$ and, intuitively, measures whether the integral curves of the fields generated by the distribution can form a mesh or not. We note that for $k \geqslant 2$ the integral curves of the vectors $\xi_{k}$ will not, in general, tangle into a web. For instance, $\xi_{1}=\partial_{y}=(0,1,0)$ and $\xi_{2}=-y \partial_{x}+\partial_{z}=(-y, 0,1)$ generate the curves of Fig. 1B, which, since do not knit a mesh, will never produce a surface. Meanwhile, the integral curves of $\xi_{1}=\partial_{y}$ and $\xi_{2}=x \partial_{x}+\partial_{z}$ form mesh surfaces (Fig. 1A) which decompose the space in layers (leaves of the foliation).
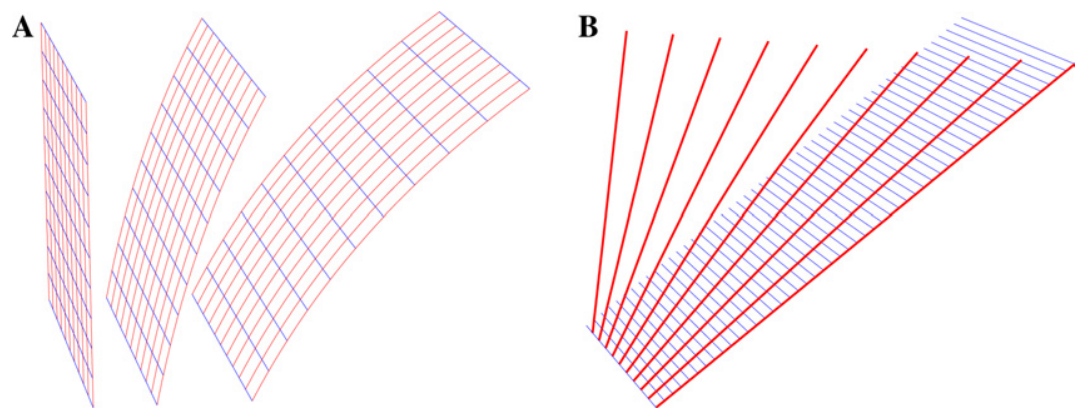

Fig. 1. Frobenius theorem: integrable (A) and nonintegrable (B) distributions. 

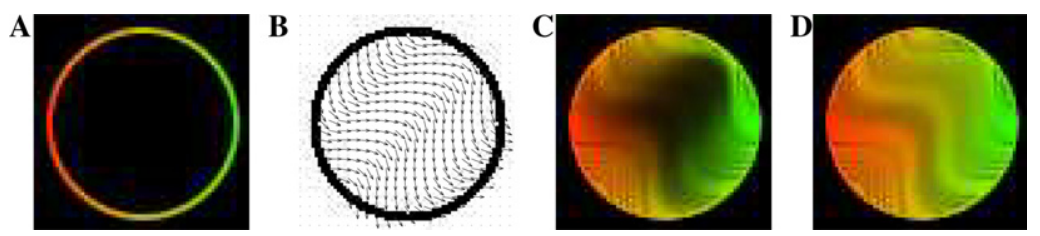

Fig. 2. General extension: function to extend (A), extension vector (B), intermediate step (C), and final extension (D).
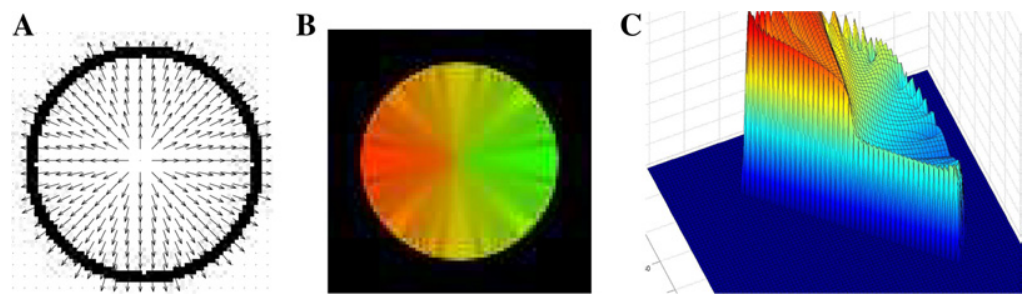

Fig. 3. Singular case: vector field (A), extension (B), and angular cut (C).

In the two dimensional case, $\partial \Omega$ is a curve, the distribution reduces to a single vector field, $\xi$, and the integrability condition is automatically satisfied. The only requirement, if we want smooth extensions, is that $\xi$ must foliate the space, that is, two different integral curves do not cross. Fig. 2 illustrates the general problem of functional extension. The function to be extended is a color map defined on the ring of Fig. 2A. The vector, $\xi$, guiding extension is the sinusoidal of Fig. 2B where the extension scope has been restricted to the area enclosed by the ring. The effect of restricting diffusion is that the final extension (Fig. 2D) changes linearly along the level lines of $\xi$. Let us show the reader that the hypothesis that $\xi$ induces a foliation on the domain is essential to guarantee convergence to smooth functions. The vector shown in Fig. 3A has a singular point at the center of the image since all its integral curves meet there. Although the extension process still exists, it converges to the sharp image of Fig. 3B, which has a jump discontinuity at the center of the image as it shows the angular cut of the mesh of Fig. 3C.

The case we will focus on is when the manifold $\partial \Omega$ is included in one of the leaves of the foliation associated to $\mathscr{D}$ and $f_{\mid \partial \Omega}=$ const, which corresponds to curve continuation and surface gap filling.

\section{Anisotropic contour closing}

We model the contour completion process as follows. Denote by $\gamma_{0}$ the set of points to connect and assume that $\xi$ is a unitary vector field defined on a band around $\gamma_{0}$ that smoothly extends its unit tangent. The anisotropic completion of contours we suggest is the extension process given by: 
A
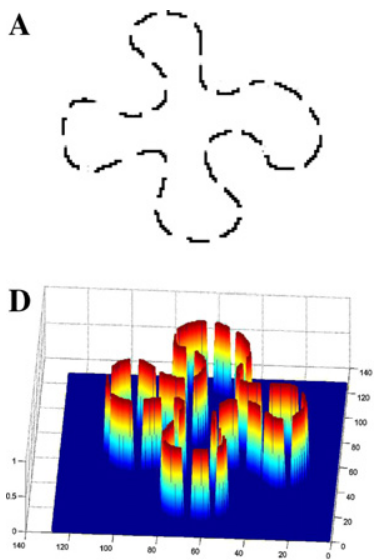

B

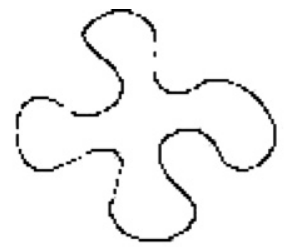

$\mathbf{E}$

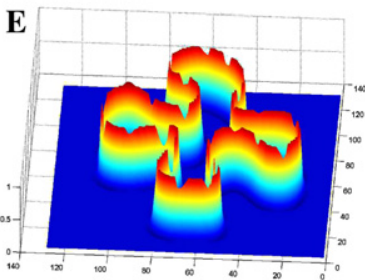

C

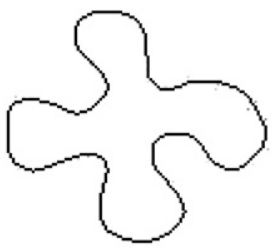

F

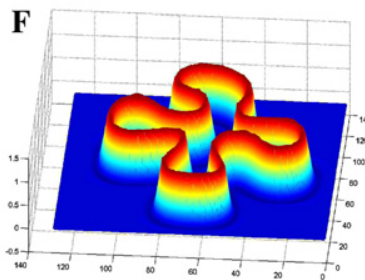

Fig. 4. Gap filling: unconnected clover (A), ridges of its mask extension (B and C), image graph of uncomplete clover (D), intermediate step (E), and closing (F).

$$
u_{t}=\operatorname{div}(\tilde{J} \nabla u) \quad \text { with } \quad u_{\mid \gamma_{0}}=u_{0}
$$

with $u_{0}$ the characteristic function of the opened contours:

$$
\chi_{\gamma_{0}}= \begin{cases}1 & \text { if } x \in \gamma_{0} \\ 0 & \text { otherwise }\end{cases}
$$

and the diffusion tensor $\tilde{J}$ a degenerate metric tensor with eigenvectors $\eta=\xi^{\perp}$ and $\xi$ and eigenvalues $\lambda_{1}=0$ and $\lambda_{2}=1$. By the properties given in Section 2, the final state will be a binary map of a closed model of the uncomplete initial contour. Intuitively, we are integrating the vector field $\xi$, that is, we are interpolating the unconnected curve segments along it. Fig. 4 illustrates the different stages in the process of gap filling for an incomplete clover (Fig. 4A). Ridges of the final characteristic function (Fig. 4F) correspond to the reconstructed complete contour (Fig. 4C).

From the above considerations, computation of an extension conforming to the image reduces to giving a smooth vector field representing its level curves.

\subsection{Coherence vector fields}

This section is devoted to the computation of an extension, $\xi$, of the unit tangent of an unconnected curve $\gamma_{0}$ smooth in a band surrounding the curve. Following the ideas presented in [8], we use the structure tensor, $S T$, upon a suitable function to compute the vector field $\xi$. The structure tensor is usually employed to determine the direction of maximum contrast change of an image $I$ in a robust way [23]. Since it is defined [13] as the mean of the projection matrices onto a regularized image gradient, the eigenvector of minimum eigenvalue, $\xi$, corresponds to the level sets unit tangent direction. Besides, since orientations do not play any role in the diffusion process, the eigenvectors of $S T$ serve to design diffusion tensors [23]. Let $I$ be an 
embedding function for $\gamma_{0}$ and note by $G_{\rho}$ a centered gaussian of variance $\rho$. Then ACC tensor is defined by:

$$
\begin{aligned}
& u_{t}=\operatorname{div}\left(Q \tilde{\Lambda} Q^{t} \nabla u\right) \\
& \text { with } Q \text { the eigenvectors of } S T_{\rho}=G_{\rho} *\left(\nabla I_{\sigma} \nabla I_{\sigma}^{T}\right), \\
& \tilde{\Lambda}=\left(\begin{array}{ll}
0 & 0 \\
0 & 1
\end{array}\right) \text { and } \nabla I_{\sigma}=G_{\sigma} * \nabla I .
\end{aligned}
$$

Since, $S T_{\rho}$ is the solution to the heat equation with initial condition the projection matrix onto the embedding function gradient, we have that $\xi$ (see scheme in Fig. 5) conforms to the Gestalt principle of good continuation (i.e., it is a infinitely differentiable field [10] that regularizes and extends $\gamma_{0}$ tangent space). The choice of the embedding function hinges upon the particular application we handle: object segmentation or disoclusion and shape restoration.

(1) Image based vector fields: In this case, $I$ is set to be the same original image or any map encoding its main features (edge, Gabor energy). This is the best choice for closing objects in real scenes with segmentation purposes, provided that level sets are differentiable curves. Fig. 6B represents ACC diffusion tensor for the golfer in Fig. 6A and the final extension Fig. 6C. In the case of object disocclusion and shape completion, original images contain no information on tangent space at $\gamma_{0}$ gaps. This forces designing $\xi$ on the grounds of one of the following Gestalt principles:

(2) Linear vector fields ( $L V F$ ): They are defined by setting the embedding function, $I$, to be the characteristic function $\chi_{\gamma_{0}}$. The associated ACC yields closed shapes that resemble the one we would get if we drew the tangent at the boundary points of the original curve and intersected the lines. Their major drawback is that if the angle between the unit tangent of two consecutive pieces is too acute the vector field becomes singular (Fig. 7A), thus yielding wrong models (Fig. 7D).

(3) Distance vector fields ( $D V F$ ): They correspond to taking $I$ to be a function representing the evolution of $\gamma_{0}$ under a monotonous geometric flow, $\gamma_{t}=\beta \vec{n}$, with $\beta>0$. That is, $I$ level sets are characterized as the set of points that are at a given distance from $\gamma_{0}$. Therefore closures (Fig. 7E) base on the principle of proximity for joining boundary points. Although this yields smooth models which are not singular at corners (Fig. 7B), in the case of a distance between contours smaller than the gap size, LVF is preferable, as DVF closed models might not conform to the shape yielded by our visual system (Fig. 7F).
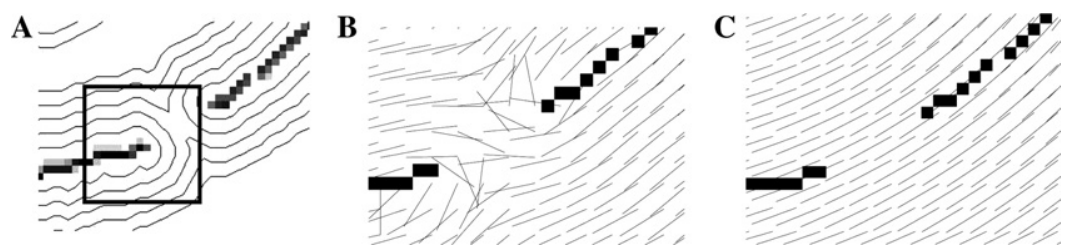

Fig. 5. Vector definition: embedding function (A), tangent space (B), and vector $\xi(\mathrm{C})$. 

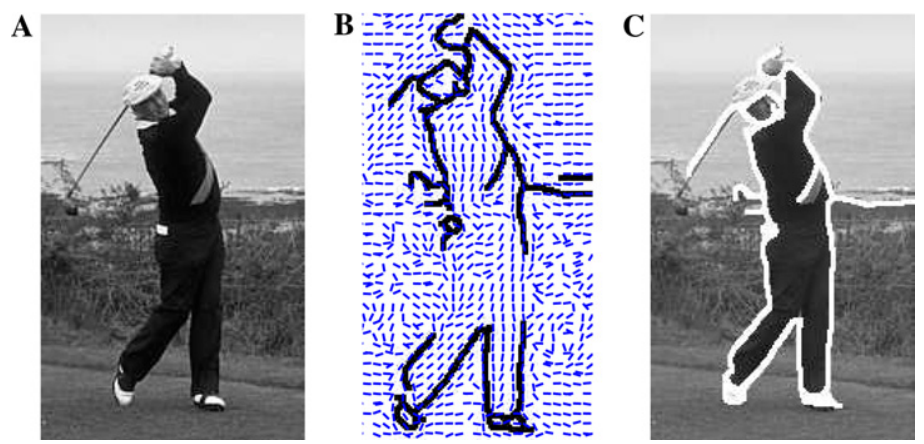

Fig. 6. ACC closing of real image: golfer (A), $\xi(\mathrm{B})$, and closure (C).
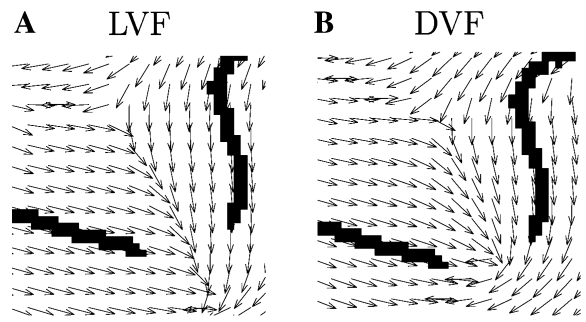

C LVF

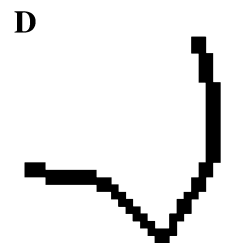

$\mathbf{E}$

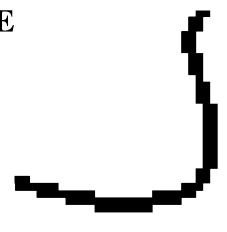

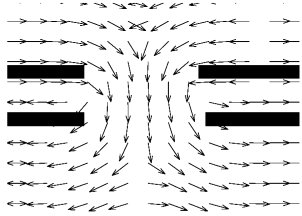

$\mathbf{F}$

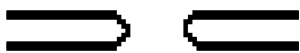

Fig. 7. Differences between LVF and DVF.

\section{Results}

To illustrate the performance of our contour closing method, two different experimental issues should be addressed. First, accuracy of shape models recovered by ACC with examples showing the practical way of choosing between DVF and LVF. Second, we present an application to object segmentation with a comparison to geodesic snakes.

\subsection{Closing contours with ACC}

In this experimental section, we will show the reliability of the shapes recovered by ACC using DVF/ LVF to guide the restricted extension process. Fig. 8 shows a set of real images (Figs. 8A-C) and a character 'S' with uniform noise added (Fig. 8D). 

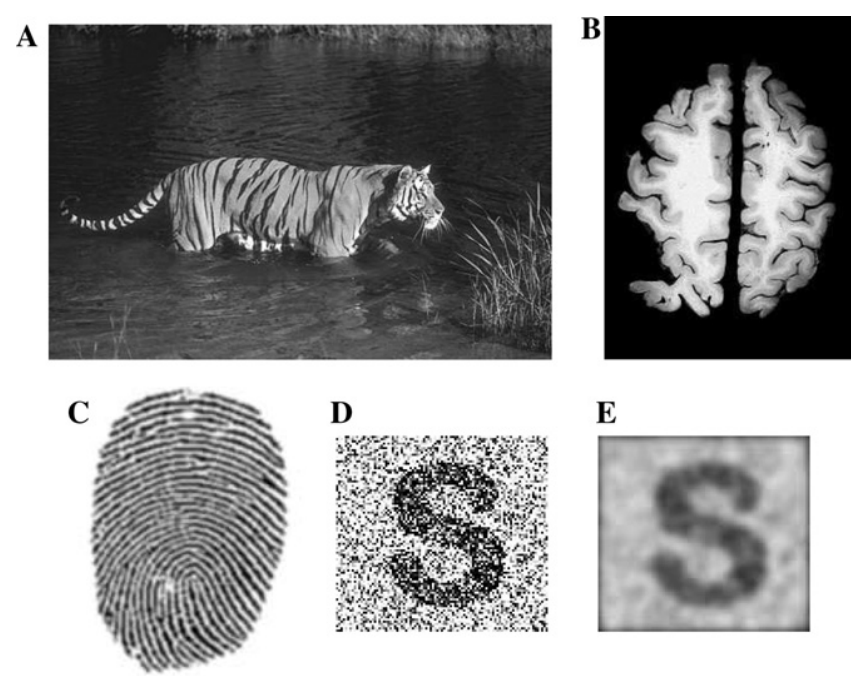

Fig. 8. Test set. Real images: tiger (A), brain (B), and fingerprint (C). Noisy images: character 'S' (D) and smoothed image $(\mathrm{F})$.

The different image features used to select points lying on the objects of interest are edges for all images but for the fingerprint, which bases on ridges. We have chosen the following standard algorithms to compute them: Canny detector for edges and curvature for ridges. The uncompleted contours in Fig. 9 have been extracted after filtering images with a gaussian of $\sigma=2$, meanwhile, in the case of the tiger (Fig. 10), we use $\sigma=4$ and select the largest segments (Fig. 10B). Contours include cases deserving special care: corner restoration (leafs in foreground plants in Fig. 10B), T-junctions (brain folds in Fig. 9A), dense lines prone to merge (fingerprint in Fig. 9C), and illusory contours (tiger tail and spine in Fig. 10B).

The numeric scheme used to compute solutions to (4) is an explicit Euler scheme for nonlinear heat equations stopped by stabilization of the speed of the evolution [10]. Scale parameters (regularization scale $\sigma$ and integration scale $\rho$ ) for the computation of Coherence Vector Fields should reach a compromise between gap filling (large scales) and separability of distinct curve structures (small scales). To such purpose we have chosen the following set of parameters: $\{\sigma=2, \rho=4\}$ for one connected component shapes (brain and ' $\mathrm{S}$ ' character), $\{\sigma=0.5, \rho=2\}$ for the tiger and $\{\sigma=0.5, \rho=1\}$ for the fingerprint. In the case of large gaps (Fig. 10B and Fig. 9B) vector fields are dynamically updated over ridges of the evolved images every 500 iterations. We have used the ridgeness measure described in [1] to compute ridges in the case of smooth contours (fingerprint and noisy ' $\mathrm{S}$ ') and Gabor energy for contours with $\mathrm{T}$-junctions and sharp corners (brain and tiger). According to the theoretic analysis given in Section 3.1, distance based vector fields properly recover a model of corners and T-junctions, meanwhile linear fields avoid merging in the contours to complete. This motivates using the distance based DVF for 
A

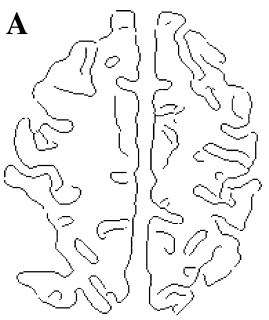

B

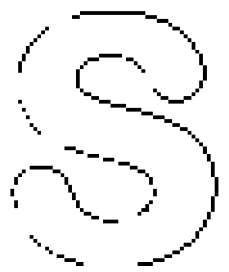

C

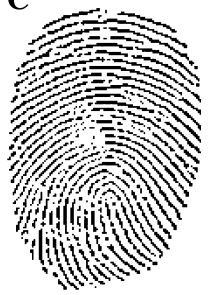

Fig. 9. Uncompleted contours.

A

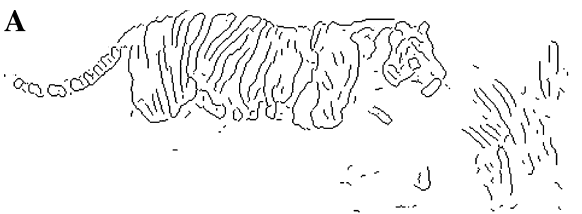

B

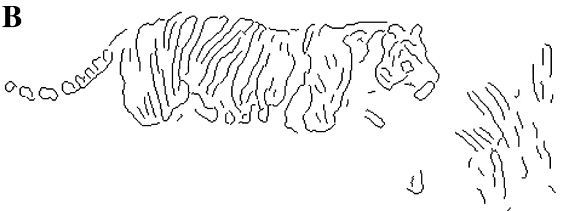

Fig. 10. Textured backgrounds: original edges (A) and largest ones (B).

restoration of contours in Figs. 9A and B, Fig. 10B and a linear LVF closing in the case of the fingerprint (Fig. 9C).

The final states achieved by ACC are the characteristic functions representing the reconstructed shapes shown in Fig. 11 and their ridges yielding contour closures are the images of Fig. 12. Since they base on distance maps, DVF closings recover shape curvatures, such as the concave part of the character ' $\mathrm{S}$ ' in Fig. $12 \mathrm{C}$, and also the acute angles of the plants leafs (Fig. 12A). On the other hand, thanks to its linearity, LVF fingerprint closure (Fig. 12D) does not merge line ends at the boundary and yields an accurate closure because of a small gap size without acute angulation.

Reliability of the restored shapes is better appreciated in the close-ups of Fig. 13, which shows three especial cases: T-junctions (Fig. 13A), illusory contours (Fig. 13B) and dense lines (Fig. 13C). T-junctions of the brain folds are properly reconstructed (Fig. 13D) because level sets of distance maps produce reliable models. Illusory contours of the tiger tail (Fig. 13E) are achieved thanks to the Gestalt principle of proximity satisfied by DVF closures. Finally, the smallest scale used to compute the linear interpolation yielded by LVF ensures that lines do not merge in the model recovered for the fingerprint (Fig. 13F).

A

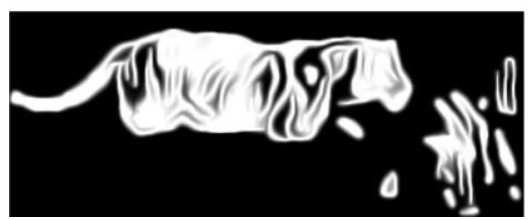

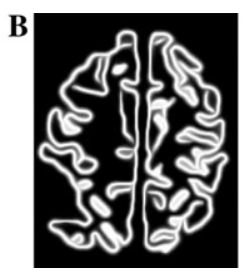
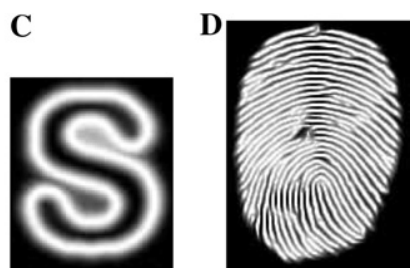

Fig. 11. Extensions of tiger (A), brain (B), 'S' (C), and fingerprint (D). 
A

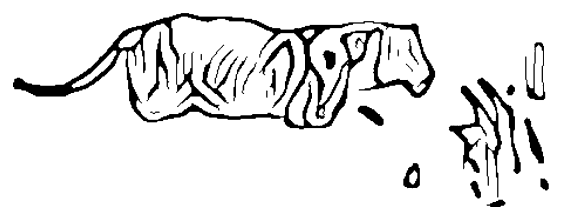

B

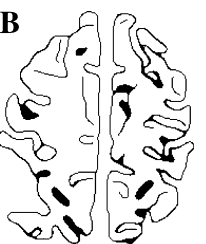

C

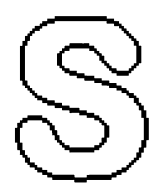

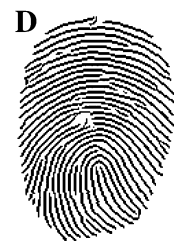

Fig. 12. Reconstructed contours using DVF (A-C) and LVF (D).

A

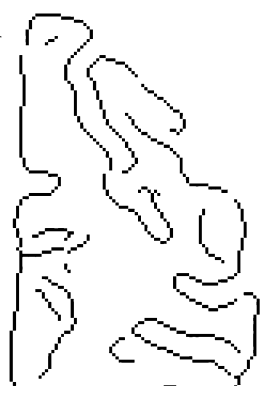

D

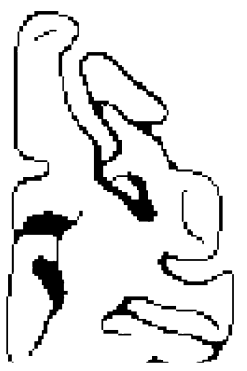

B

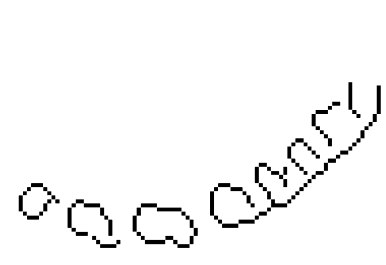

$\mathbf{E}$

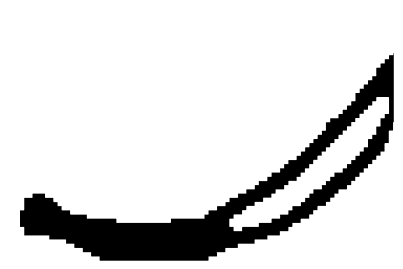

C

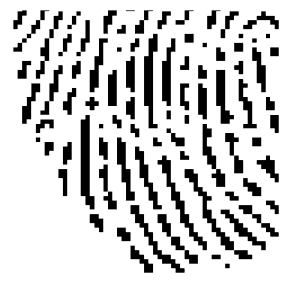

F

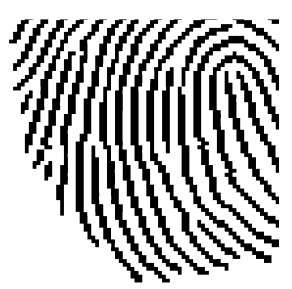

Fig. 13. Restoration of T-junctions (D), of brain folds (A), illusory contours (E), of tiger tail (B), and recovery of the dense lines $(\mathrm{F})$, and of the fingerprint $(\mathrm{C})$.

\subsection{Application to object segmentation}

Smooth shape representation plays an important role in image segmentation. Connecting a set of points that lie on the object of interest, whatever its geometry, is still an open question. Parametric snakes [15] and geodesic snakes [3,4] are the two techniques most commonly used by the image processing community. On one hand, in spite of yielding smooth models, poor convergence to concave shapes limits classic snakes applicability. On the other hand, geodesic snakes convergence to multiple objects, does not compensate for their slow convergence to piece wise linear curves prone to penetrate into contours large gaps. We argue that the framework of classic snakes provides with an efficient way of shape modelling, both in terms of computational time and compact representation of a reliable model of the shape. The segmenting strategy we propose is the following. 


\subsubsection{The segmenting strategy}

We base image object segmentation on the approximation of a set of (possibly unconnected) points that conform to given characteristics exclusive to the contour of the object we want to model. We consider that the object is successfully segmented once we have a closed contour approaching this set of points. We propose the following strategy to model uncompleted contours. First, we apply functional extension using ACC to the selected set of points to produce a closed contour. Ridges of the final extension are the curve of level zero of the curvature distance map introduced in [9]. This convergent map [10] bases on using the mean curvature flow to propagate the zero level curve. For the outward propagation, we evolve the initial shape under the flow given by negative curvature (5) until it reaches the stable convex shape and, then, use an outward Euclidean distance map. For the inward propagation we use evolution under positive curvature (5) until the curve becomes circular and then use the Euclidean distance map to this circle to complete it. The gradient of this map is called curvature vector flow (CVF).

Evolution by negative curvature Evolution by positive curvature

$$
\gamma_{t}=\min ((\kappa, 0)) \vec{n}, \quad \gamma_{t}=\max ((\kappa, 0)) \vec{n} .
$$

\subsubsection{Segmentation of real images}

We devote this last experiment to assessment of the segmenting capabilities of the proposed strategy in comparison to geodesic snakes. We have used standard formulations:

Geodesic evolution : $\gamma_{t}=\left(\alpha g(|\nabla I|)\left(V_{0}+\kappa\right)-(1-\alpha)\langle\nabla g(|\nabla I|), \vec{n}\rangle\right) \vec{n}$,

Parametric energy : $E(\gamma)=\int_{\gamma}\left(\alpha\left\|\gamma^{\prime}\right\|^{2}+\beta\left\|\gamma^{\prime \prime}\right\|^{2}+E_{\text {ext }}\right) \mathrm{d} u$.

The set of test images (a deer, a horse, and a golfer in Fig. 14) include textured backgrounds (grass and ground in the deer and horse images) and weak edges (golfer legs). To minimize the impact of textured backgrounds, edges (Fig. 14) have been extracted using the visual inhibition approach given in [11] and a curve length filtering. The closed contours used to compute CVF (overimpressed in Fig. 15) result from interpolating the largest edges. Geodesic snake metric has been computed with

A

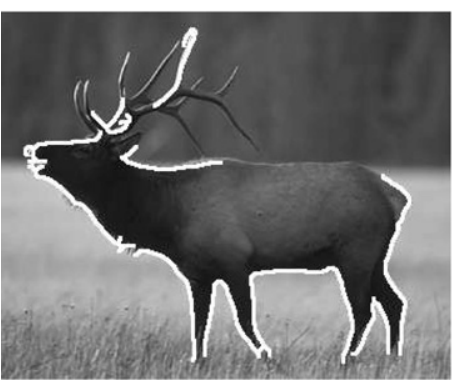

B

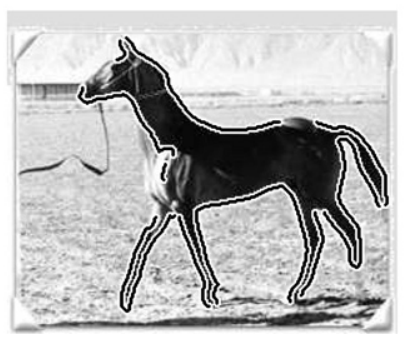

C

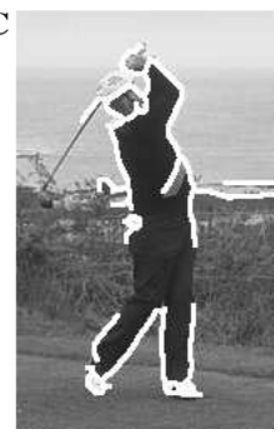

Fig. 14. Set of real images to segment with their edges. 
A

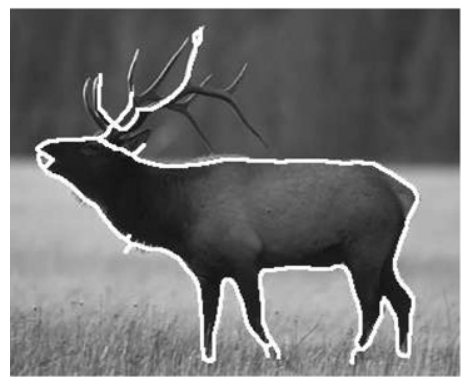

B

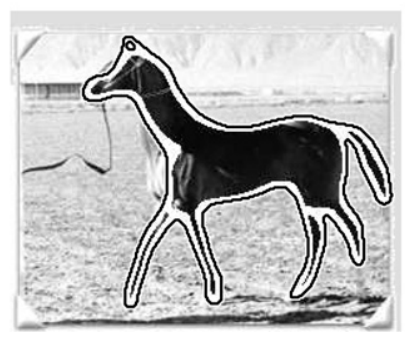

C

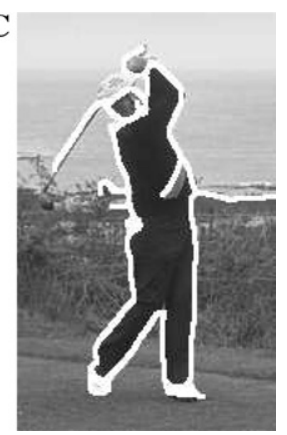

Fig. 15. ACC closure.

$\sigma=2$ for the golfer and $\sigma=4$ in the case of the deer and horse. Because such scales are prone to yield open image edges in the metric $g$, the value of $V_{0}$ was chosen to avoid penetrating into contours $\left(\left\{V_{0}=0.1, \alpha=0.3\right\}\right)$. For parametric snakes the parameter set was tuned to yield smooth curves, $\{\alpha=0.3, \beta=0.6\}$. Final results of the segmentation are shown in Fig. 16 for geodesic snakes and Fig. 17 for CVF-snakes: dashed contours are the initial snakes and solid ones the final models.

On one side, convergence of geodesic snakes is only guaranteed for almost texture-free backgrounds like the deer in Fig. 16A. In the presence of more prominent texture in image background (horse) geodesic snakes fail to stabilize at the right boundaries, even if they are initialized close to the contour of interest. In the case of the horse (Fig. 16B) the snake still enters one of the back legs and, though a close initialization, the edge due to the shadow (bottom) traps the snake so it fails to adapt to the horse belly. In spite of using a low $\sigma$ for the golfer (Fig. 16C), the geodesic model has swallowed both legs because their contrast change is not strong enough. On the other side, because segmentations only rely on the accuracy of the closed models given by ACC ( $\mathrm{CVF}$ guarantees snake convergence), the overall performance is better than the geodesic model. The deer model (Fig. 17A) is smoother than its geodesic counterpart. In the case of the horse (Fig. 17B), our model perfectly captures its legs, tail and belly, yielding a smooth silhouette, thanks to a high $\beta$ (curva-

A

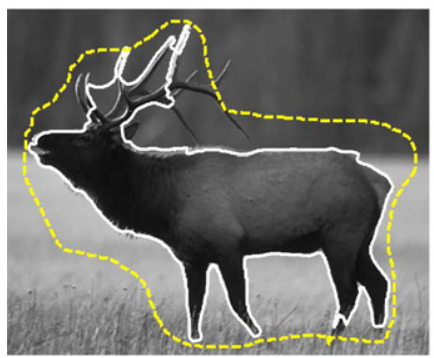

B

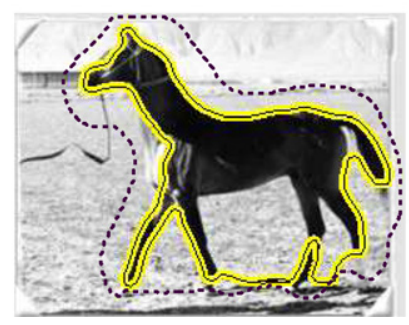

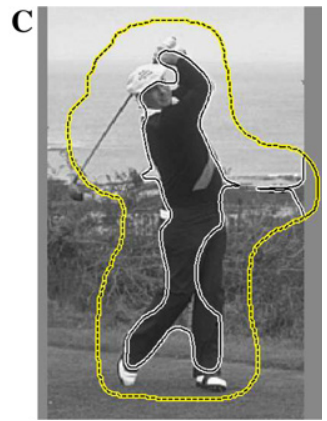

Fig. 16. Initial and final geodesic snakes. 
A

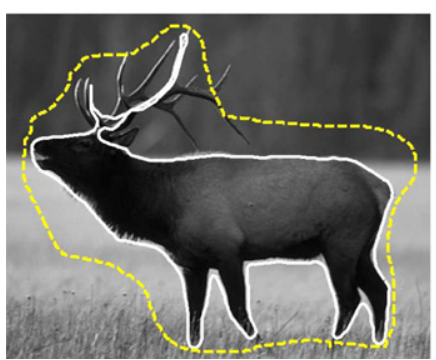

B

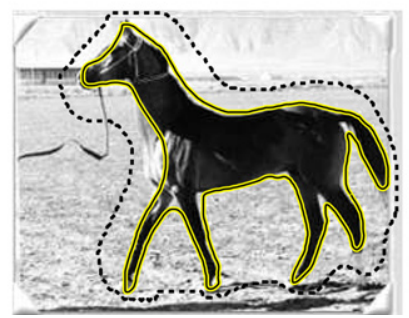

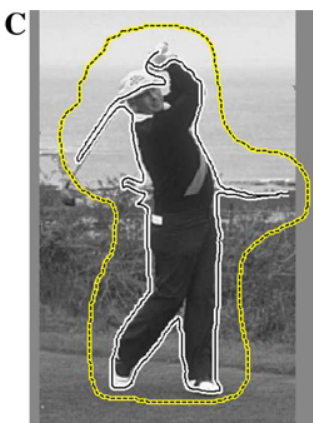

Fig. 17. Initial and final CVF-snakes.

ture) term. In the case of the golfer (Fig. 17A), ACC closure guarantees the right capture of the legs weak edges and stick.

\section{Conclusions}

This paper addresses the issue of contour completion oriented to object segmentation. Completion of contours is considered as a particular case of functional extension driven by a diffusion tensor that restricts to some curves of the image domain. In this context, we present a novel approach to diffusion processes from the point of Riemmanian geometry. Classic results on differential geometry yield sufficient conditions for the unique existence of solutions to a restricted anisotropic extension/diffusion process. The fact that a smooth vector field automatically satisfies the former conditions reduces contour completion to the definition of a vector field representing the tangent of the contours to be closed. For a reliable and efficient computation of the latter, we suggest using the structure tensor to define vector extensions conforming with tangents of unconnected contours at gap boundaries (principle of good continuation). The segmenting strategy is a combination of ACC closing and a distance map based on the evolution of closed contours by $\mathrm{min} / \mathrm{max}$ curvature introduced in [9]. Based on the grounds that a distance map represents the evolution of an initial curve in time under a geometric flow, it tracks evolution by mean curvature flow to avoid shocks. The fact that the geometry of the initial curve is taken into account, makes snake convergence robust whatever the concavity of the zero level curve.

Experiments illustrate accuracy of shape models obtained by ACC and its capability of handling corner, T-junction and illusory contours. Segmentation of real images show that our strategy surpasses parametric snakes performance and is competitive with geodesic snakes, performing even better in textured backgrounds.

\section{References}

[1] A.M. López, D. Lloret, J. Serrat, J.J. Villanueva, Multilocal creaseness based on the level-set extrinsic curvature, Comp. Vis. Image Understand. 77 (2000) 111-144. 
[2] C. Ballester, M. Bertalmío, V. Caselles, G. Sapiro, J. Verdera, Filling-in by joint interpolation of vector fields and gray levels, IEEE Trans. Image Process. (2001).

[3] V. Caselles, F. Catte, T. Coll, F. Dibos, A geometric model for active contours, Numer. Math. 66 (1993) 1-31.

[4] V. Caselles, R. Kimmel, G. Sapiro, Geodesic active contours, Int. J. Comp. Vision 22 (1) (1997) 61-79.

[5] T. Chan, J. Shen, Non-texture inpaintings by curvature driven diffusions, J. Vis. Commun. Image Rep. 12 (4) (2001) 436-449.

[6] E.B. Davies, Heat Kernels and Spectral Theory, Cambridge Tracts. in Math. 92, Cambridge University Press, 1989.

[7] L.C. Evans, Partial differential equations, Berkeley Math. Lect. Notes. 3B (1993).

[8] D. Gil, P. Radeva, F. Vilariño, Anisotropic Contour Completion, ICIP, 2003.

[9] D. Gil, P. Radeva, Curvature vector flow to assure convergent deformable models, EMMCVP, 2003.

[10] D. Gil, Geometric Differential Operators for Shape Modelling. PhD Thesis, 2004, on-line document at Available from: <http://www.cvc.uab.es/ debora/ $>$.

[11] C. Grigorescu, N. Petkov, M.A. Westenberg, Contour perception based on non-classical receptive field inhibition, IEEE Trans. Imag. Proc. 12 (2003) 7.

[12] L. Williams, D. Jacobs, Stochastic completion fields: a neural model of illusory contour shape and salience, ICCV' 95, 1995.

[13] B. Jähne, Spatio-temporal image processing. Lecture Notes in Comp. Science, vol. 751, Springer, Berlin, 1993.

[14] G. Kanizsa, Organization in Vision: Essays in Gestalt Continuation, Praeger, New York, 1979.

[15] M. Kass, A. Witkin, D. Terzopoulos, Snakes: active contour models, J. Comp. Vision 1 (1987) 321-331.

[16] S. Lang, Linear Algebra, second ed., Addison Wesley, 1971.

[17] S. Masnou, J.M. Morel, Level Lines Based Disocclusion, in: Proc. IEEE Int. Conf. on Image Process. Chicago IL, 1998, pp. 259-263.

[18] M. Nitzberg, D. Mumford, T. Shiota, Filtering Segmentation and Depth, Springer-Verlag, Berlin, 1993.

[19] N. Paragios, O. Mellina-Gottardo, V. Ramesh, Gradient vector flow fast geodesic active contoursIEEE ICCV, vol. I, Vancouver, Canada, 2001.

[20] J.A. Sethian, Level Set Methods: Evolving Interfaces in Geometry, Fluid Mechanics, Computer Vision and Material Sciences, Cambridge University Press, 1996.

[21] M. Spivak, A Comprehensive Introduction to Differential Geometry, Publish or Perish cop., Houston, 1979.

[22] C. Xu, J.L. Prince, Generalized gradient vector flow external forces for active contours, Signal Process. An Int. J. 71 (2) (1998) 132-139.

[23] J. Weickert, Anisotropic Diffusion in Image Processing, PhD Thesis, 1996. 